\title{
Razões motivacionais relacionadas à prática de atividade física em idosos - Estudo piloto para subsidiar estratégias na atenção primária à saúde do município de Caicó
}

\author{
Motivational reasons related to the practice of physical activity in the elderly - Pilot study to \\ support strategies in primary health care in the municipality of Caicó \\ Razones motivacionales relacionadas con la práctica de actividad física en adultos mayores - \\ Estudio piloto de apoyo a estrategias en atención primaria de salud en el municipio de Caicó
}

Recebido: 03/05/2021 | Revisado: 11/05/2021 | Aceito: 14/06/2021 | Publicado: 26/06/2021

\author{
Thiago Santos de Araújo \\ ORCID: https://orcid.org/0000-0001-9877-6218 \\ Universidade Federal do Rio Grande do Norte, Brasil \\ E-mail: thiagosantos82@ hotmail.com \\ Ana Carolina Patrício de Albuquerque Sousa \\ ORCID: https://orcid.org/0000-0001-7517-8792 \\ Universidade Federal do Rio Grande do Norte, Brasil \\ E-mail: acapas@gmail.com
}

\begin{abstract}
Resumo
Ao entrarmos no século XXI, o envelhecimento populacional causou um aumento das demandas sociais e econômicas, e em todo o mundo, a proporção de pessoas com 60 anos ou mais cresce mais rapidamente que a de qualquer outra faixa etária. A atividade física é uma importante estratégia para promoção da saúde e traz inúmeros benefícios, dentre eles: maior longevidade, redução das taxas de morbidade e mortalidade, melhora na capacidade fisiológica em portadores de doenças crônicas, além de benefícios psicológicos. Com base nessas perspectivas, este estudo tem como objetivo investigar as razões motivacionais e os possíveis fatores relacionados à prática de atividade física em idosos, e torna-se relevante pelo fato de, no Brasil, ainda serem escassos estudos abordando as motivações que levam idosos a praticarem atividade física regularmente. Trata-se de um estudo piloto, caracterizado como individuado, observacional e transversal. Os dados foram coletados em Unidades Básicas de Saúde da Atenção Primária do município de Caicó (RN) de março a abril de 2020. Esse estudo contou com a participação de 30 indivíduos com 60 anos ou mais, a maioria do sexo feminino, e praticantes de atividade de intensidade leve ou moderada. As comorbidades mais frequentes foram: cardiopatias, hipertensão e doenças pulmonares. As razões motivacionais que levam os idosos a praticarem atividade física foram relacionadas à saúde, sociabilidade e controle do estresse, sendo menos motivada pela estética e competitividade.
\end{abstract}

Palavras-chave: População idosa; Atenção primária à saúde; Atividade física; Motivação.

\begin{abstract}
As we enter the 21st century, population aging has caused an increase in social and economic demands, and worldwide, the proportion of people aged 60 and over grows faster than that of any other age group. Physical activity is an important strategy for health promotion and brings numerous benefits, among them: greater longevity, reduced rates of morbidity and mortality, improved physiological capacity in patients with chronic diseases, in addition to psychological benefits. Based on these perspectives, this study aims to investigate the motivational reasons and possible factors related to the practice of physical activity in the elderly, and it becomes relevant because, in Brazil, there are still few studies addressing the motivations that lead elderly people to practice physical activity regularly. This is a pilot study, characterized as individualized, observational and cross-sectional. The data were collected in Basic Health Units of Primary Care in the municipality of Caicó (RN) from March to April 2020. This study involved the participation of 30 individuals aged 60 years or older, most of them female, and practitioners of light or moderate intensity activity. The most frequent comorbidities were: heart disease, hypertension and lung diseases. The motivational reasons that lead the elderly to practice physical activity were related to health, sociability and stress control, being less motivated by aesthetics and competitiveness.
\end{abstract}

Keywords: Elderly population; Primary health care; Physical activity; Motivation.

\section{Resumen}

Al entrar en el siglo XXI, el envejecimiento de la población ha provocado un aumento de las demandas sociales y económicas, y en todo el mundo, la proporción de personas de 60 años o más crece más rápido que la de cualquier otro grupo de edad. La actividad física es una estrategia importante para la promoción de la salud y aporta numerosos 
beneficios, entre ellos: mayor longevidad, menores tasas de morbilidad y mortalidad, mejora de la capacidad fisiológica en pacientes con enfermedades crónicas, además de beneficios psicológicos. Con base en estas perspectivas, este estudio tiene como objetivo investigar las razones motivacionales y los posibles factores relacionados con la práctica de actividad física en los ancianos, y adquiere relevancia porque, en Brasil, aún existen pocos estudios que aborden las motivaciones que llevan a los ancianos a practicar. actividad física con regularidad. Se trata de un estudio piloto, que se caracteriza por ser individualizado, observacional y transversal. Los datos fueron recolectados en Unidades Básicas de Salud de Atención Primaria del municipio de Caicó (RN) de marzo a abril de 2020. Este estudio contó con la participación de 30 personas de 60 años o más, la mayoría mujeres, y practicantes de leve o moderada actividad de intensidad. Las comorbilidades más frecuentes fueron: cardiopatía, hipertensión y enfermedades pulmonares. Las razones motivacionales que llevan a las personas mayores a la práctica de actividad física se relacionan con la salud, la sociabilidad y el control del estrés, estando menos motivados por la estética y la competitividad.

Palabras clave: Población de ancianos; Atención primaria de salud; Actividad física; Motivación.

\section{Introdução}

Um dos maiores feitos da humanidade foi a ampliação do tempo de vida, que se fez acompanhar da melhora substancial dos parâmetros de saúde das populações, ainda que essas conquistas estejam longe de se distribuir de forma equitativa, em diferentes países e contextos socioeconômicos. Chegar à velhice se transformou em uma das maiores conquistas do século XX, no entanto, tornou-se o grande desafio para o século atual (Veras \& Oliveira, 2018). De acordo com Lima e Delgado (2017) o envelhecimento pode ser conceituado como um conjunto de modificações morfológicas, fisiológicas, bioquímicas e psicológicas, que determinam a perda progressiva da capacidade de adaptação do indivíduo ao meio ambiente, sendo considerado um processo dinâmico e progressivo. Segundo o Instituto Brasileiro de Geografia e Estatística do Brasil (2019), o Brasil é um país com muitos idosos, gerando assim grandes demandas para políticas públicas de saúde para atender esse público. Considera-se que ao passar dos 60 anos de idade a pessoa necessitará de uma série de cuidados que poderá favorecer o prolongamento de vida com saúde, autonomia e disposição. No Brasil, estima-se que, em 2025, o país ocupará o sexto lugar entre os países com maior população idosa, 25 milhões de pessoas, e que, em 2050, atingirá marca superior a 51 milhões de idosos (IBGE, 2019).

Atualmente, a Organização Panamericana de Saúde (2019) conclamou os países da Região das Américas a fortalecerem seus sistemas de saúde e de proteção social para responderem às demandas de cuidados de longa duração que, em quantidade, triplicarão nas próximas três décadas, passando de oito milhões a 27 ou a 30 milhões até 2050. No mesmo sentido, a Organização dos Estados Americanos (2015), em 15 de junho de 2015, já havia aprovado a Convenção Interamericana sobre a Proteção dos Direitos Humanos da Pessoa Idosa, por meio de um documento juridicamente vinculante. Um item especifico dispõe que os "Estados Partes devem adotar medidas para desenvolver um sistema integral de cuidados que leve em conta a perspectiva de gênero e o respeito à dignidade e integridade física e mental do idoso, para lhe garantir o gozo efetivo de seus direitos humanos por meio de cuidados de longo prazo". Os estudiosos Fleck e Kraemer (2017) garantem que mesmo os idosos em idade bem avançadas podem se beneficiar com a prática de exercícios físicos, que é capaz de melhorar a saúde e a capacidade funcional, levando a aumentos significativos na qualidade de vida. Farias Júnior (2015) afirmam que as atividades físicas agem retardando os problemas causados ao organismo pelo processo de envelhecimento, indicando que devemos estimular a fuga do sedentarismo a fim de melhorar a capacidade funcional, autoestima e prevenir doenças degenerativas.

Pode-se considerar que as diversas teorias psicológicas e de comportamento humano podem nortear as motivações para adoção de hábitos saudáveis por parte da população idosa, contribuindo significativamente para a base de conhecimentos que podem guiar o desenvolvimento de novas intervenções para promover a aceitação e manutenção da prática de atividade física. A adoção de hábito e a adesão à prática de atividade física pelos idosos podem estar relacionados e motivados a fatores como: promoção da saúde, bem-estar, gosto pela atividade física, socialização, entre outros (Eiras, Silva, Souza \& Vendruscolo, 2010). 
Cabe destacar o papel da Atenção Primária à Saúde (APS) que serve de base para um novo modelo de assistência e organização dos sistemas de saúde, configurando-se como porta de entrada prioritária e capaz de prestar serviço para todas as pessoas e famílias da comunidade, que também dele participam ativamente. A Estratégia Saúde da Família (ESF), por sua vez, configura-se como uma reorientação do modelo assistencial em saúde na APS que busca garantir a integralidade da assistência centrada na família e direcionada à comunidade. Pelos idosos representarem uma das parcelas mais vulneráveis da população, portanto, espera-se que os serviços de APS sejam capazes de se organizar e ofertar serviços aos idosos considerando suas demandas específicas e busca pela redução das iniquidades em saúde (Brasil, 2012).

Dessa forma, acredita-se que a prática regular de atividades físicas deve ser estimulada por propostas de políticas públicas, a partir da APS, favorecendo uma atenção integral ao indivíduo que está em processo de envelhecimento. Partindo dessa premissa, o presente estudo teve como objetivo investigar as razões motivacionais e os possíveis fatores relacionados à prática de atividade física em idosos, atendidos pela APS, auxiliando no planejamento e direcionamento de estratégias metodológicas e estruturais dos programas de atividade física para idosos.

\section{Metodologia}

O presente estudo é do tipo piloto, caracterizado como individuado, observacional, transversal e abordagem quantitativa. A pesquisa foi realizada em 3 unidades básicas de saúde (UBS's) do município de Caicó, Rio Grande do Norte (RN). Caicó é um município brasileiro pertencente ao estado do RN, localizado na zona central do estado, distante $282 \mathrm{~km}$ da capital de estado, Natal.

A população do estudo foi constituída por indivíduos com 60 anos ou mais, atendidos pela APS da zona urbana do município de Caicó. De acordo com o último IBGE (2020), o município de Caicó possui, aproximadamente, 7.716 pessoas com 60 anos ou mais, considerando a população urbana e rural (https://cidades.ibge.gov.br/). Além disso, sabe-se que o município apresenta 21 UBS's com 95\% de abrangência populacional.

A amostra foi restrita a 3 UBS's da zona leste do município de Caicó, sendo representativo apenas para um contexto restrito da zona leste do município de Caicó, porém, podendo ser reproduzido em outros contextos. Os critérios de elegibilidade foram: idosos com 60 anos ou mais, de ambos os sexos; comunitários cadastrados nas Unidades Básicas de Saúde da zona urbana do município; não estar temporária ou permanentemente acamado; ser encontrado para avaliação em no máximo três tentativas, durante o recrutamento; conseguir concluir todas as etapas da coleta dos dados.

Os dados foram coletados no período de fevereiro a março de 2020, por uma equipe devidamente treinada para aplicar os instrumentos de coleta. Os participantes foram convidados a participar da pesquisa e assinar o Termo de Consentimento Livre Esclarecido (TCLE). As informações coletadas foram referentes a: características sociodemográficas, dados antropométricos, condições de saúde, prática de atividade física e razões motivacionais para prática de atividade física.

Quanto às características sociodemográficas, foram coletadas as seguintes informações: sexo, idade, estado civil, o exercício ou não de trabalho remunerado e grau de escolaridade. Foram tomadas medidas antropométricas da estatura, peso corporal, circunferências da cintura e do quadril. $\mathrm{Na}$ aferição da estatura, foi utilizado um estadiômetro transportável, da marca SANNY®, com $212 \mathrm{~cm}$ e precisão de $0,1 \mathrm{~cm}$, solicitando que os idosos tirassem os calçados e se colocassem de costas para o estadiômetro, em posição ereta e com os pés unidos (Charro, 2020). Na verificação do peso corporal, foi utilizada uma balança digital portátil da marca SPEEDO $®$, com capacidade de $150 \mathrm{Kg}$ e com precisão de $100 \mathrm{~g}$. Pediu-se que as mesmas retirassem os calçados e objetos, instruiu-se para que ficassem em pé sobre a plataforma da balança, com afastamento lateral dos pés, eretos e com olhar fixo para a frente (Charro, 2020). A medida da circunferência da cintura, utilizou-se uma fita métrica flexível com precisão de $1 \mathrm{~mm}$, da marca SANNY®, e a medição foi realizada com o idoso em pé, circundando-se a fita na linha natural da cintura, na região mais estreita entre o tórax e o quadril, no ponto médio entre a última costela e a crista ilíaca (Charro, 2020). 
Na circunferência do quadril, utilizou-se uma fita métrica flexível com precisão de $1 \mathrm{~mm}$, da marca SANNY®, realizada o idoso em pé, pernas unidas, circundando-se a fita na linha natural do quadril, na região mais volumosa dos glúteos (Charro, 2020).

Utilizou-se, para a análise do Índice de Massa Corporal (IMC), calculado a partir da divisão da massa corporal em quilogramas pela estatura elevada ao quadrado $\left(\mathrm{kg} / \mathrm{m}^{2}\right)$ e os pontos de corte recomendados pelo Ministério da Saúde (Brasil, 2004) para avaliação da população idosa. Com base no IMC para idosos, foram considerados baixo-peso o IMC $\leq 22$, eutrófico $22>\mathrm{IMC}>27$ e sobrepeso o IMC $\geq 27 \mathrm{~kg} / \mathrm{m}^{2}$ (Who, 2000).

A verificação do risco de desenvolvimento de complicações associadas à obesidade, através da relação das circunferências da cintura e do quadril (IRCQ), tomando por base os valores sugeridos por sexo pela Organização Mundial da Saúde (Who, 2008) que considera que, para mulheres, valores acima de 0,82 representam um risco aumentado, e para homens e acima de 0,94. A condição de saúde foi avaliada a partir da autopercepção da saúde dos idosos. A autopercepção de saúde foi avaliada perguntando ao idoso, a partir de sua concepção subjetiva, como ele considera sua saúde, cuja resposta varia entre muito boa, boa, regular, ruim ou muito ruim.

Além disso, com a finalidade de responder a problemática deste estudo e atender aos objetivos traçados, foi utilizado o instrumento denominado IMPRAF-54, validado para diferentes faixas etárias, desde adolescentes a idosos (Alpha de Cronbach superior a 0,82 em todas as dimensões avaliadas), tem a proposição de medir as seis dimensões (controle de estresse, saúde, sociabilidade, competitividade, estética e prazer) associadas à motivação para a prática regular de atividades físicas (Balbinotti, 2011). O questionário é constituído por 54 itens, agrupados em 9 blocos e que são, de forma individual, avaliados por meio de uma escala Likert de 5 pontos, "isso me motiva pouquíssimo" a "isso me motiva muitíssimo". Por meio desta escala são identificadas as dimensões que são consideradas mais motivadoras para a prática de atividade física.

A presença de comorbidades foi avaliada através do autorrelato, considerando o diagnóstico médico no último ano, ou uso contínuo de medicamentos para o tratamento das seguintes doenças crônicas: cardiopatias, hipertensão arterial, diabetes mellitus, artrite ou reumatismo, doenças pulmonares, depressão, osteoporose e acidente vascular encefálico.

Os dados foram tabulados e armazenados e processados no software estatístico Statistical Package for the Social Science versão 20.0 para Windows (SPSS 20.0). A análise estatística constou de análise descritiva (frequências, medidas de tendência central e medidas de dispersão) para caracterizar a amostra quanto às características sociodemográficas, às condições de saúde e os hábitos de vida. Em seguida, para uma melhor compreensão da motivação para a prática de atividade física, foi realizada uma análise bivariada (ANOVA) para comparação das médias dos escores brutos das dimensões em relação a variáveis independentes de condições de saúde.

O estudo foi aprovado pelo Comitê de Ética em Pesquisa da Faculdade de Ciências da Saúde (CEP FACISA/UFRN), conforme as determinações da Resolução n ${ }^{\circ}$ 466/12 do Conselho Nacional de Saúde (CNS), que define as diretrizes e normas regulamentadoras que regem a pesquisa envolvendo seres humanos, conforme as suas recomendações, tendo registro no CAAE: 24485819.7.0000.5568 e Número do Parecer: 3.737.785.

\section{Resultados}

Ao todo este estudo pesquisou 30 idosos de 60 anos ou mais atendidos pelas mais diversas Unidades Básicas de Saúde da APS do município de Caicó no período de março a abril de 2020. A tabela I apresenta a amostra composta em sua maioria pelo sexo feminino $(86,7 \%)$, com média de idade de 71,6 $\pm 6,3$ anos, estado civil casado $(36,7 \%)$, escolaridade inferior ao $1^{\circ}$ grau completo $(63,3 \%)$ e sem exercer trabalho remunerado $(80 \%)$. Quanto à condição e concepção de saúde os entrevistados responderam ter uma saúde excelente (3,3\%), muito boa (23,3\%), boa (20\%), regular (43,3\%) e ruim (10\%); e quanto aos hábitos de vida, a maioria dos indivíduos relataram não fumar $(83,3 \%)$ e não ser etilistas $(93,3 \%)$. 
A Tabela 1 apresenta a relação das variáveis antropométricas da amostra quanto a estatura, peso corporal, circunferência da cintura, do quadril, IMC e IRCQ, observou-se que a estatura da amostra pesquisada variou entre $1,33 \mathrm{~m}$ e 1,70m; o peso corporal variou entre $36 \mathrm{~kg}$ e $91,7 \mathrm{~kg}$; a circunferência da cintura variou entre $62 \mathrm{~cm}$ e $118 \mathrm{~cm}$; a circunferência do quadril esteve entre $80 \mathrm{~cm}$ e $125 \mathrm{~cm}$. De acordo com o IMC, a amostra apresentou resultados onde, $40 \%$ dos idosos foram considerados eutróficos, $40 \%$ com sobrepeso e $20 \%$ com baixo peso; percebe-se ainda que em relação desenvolvimento de complicações associadas à obesidade (IRCQ), os valores do IRCQ para o sexo feminino há predominância de um IRCQ de Alto Risco, enquanto no sexo Masculino uma predominância de um IRCQ de Médio Risco. Ao tocante à pratica de atividade física, a maior parte dos entrevistados relataram praticar atividade regularmente (73,3\%); sendo que a atividade física de intensidade leve foi a mais relatada $(43,3 \%)$.

Tabela 1: Caracterização da amostra quanto a: características sociodemográficas, condições de saúde e hábitos de vida. Caicó (RN). 2020.

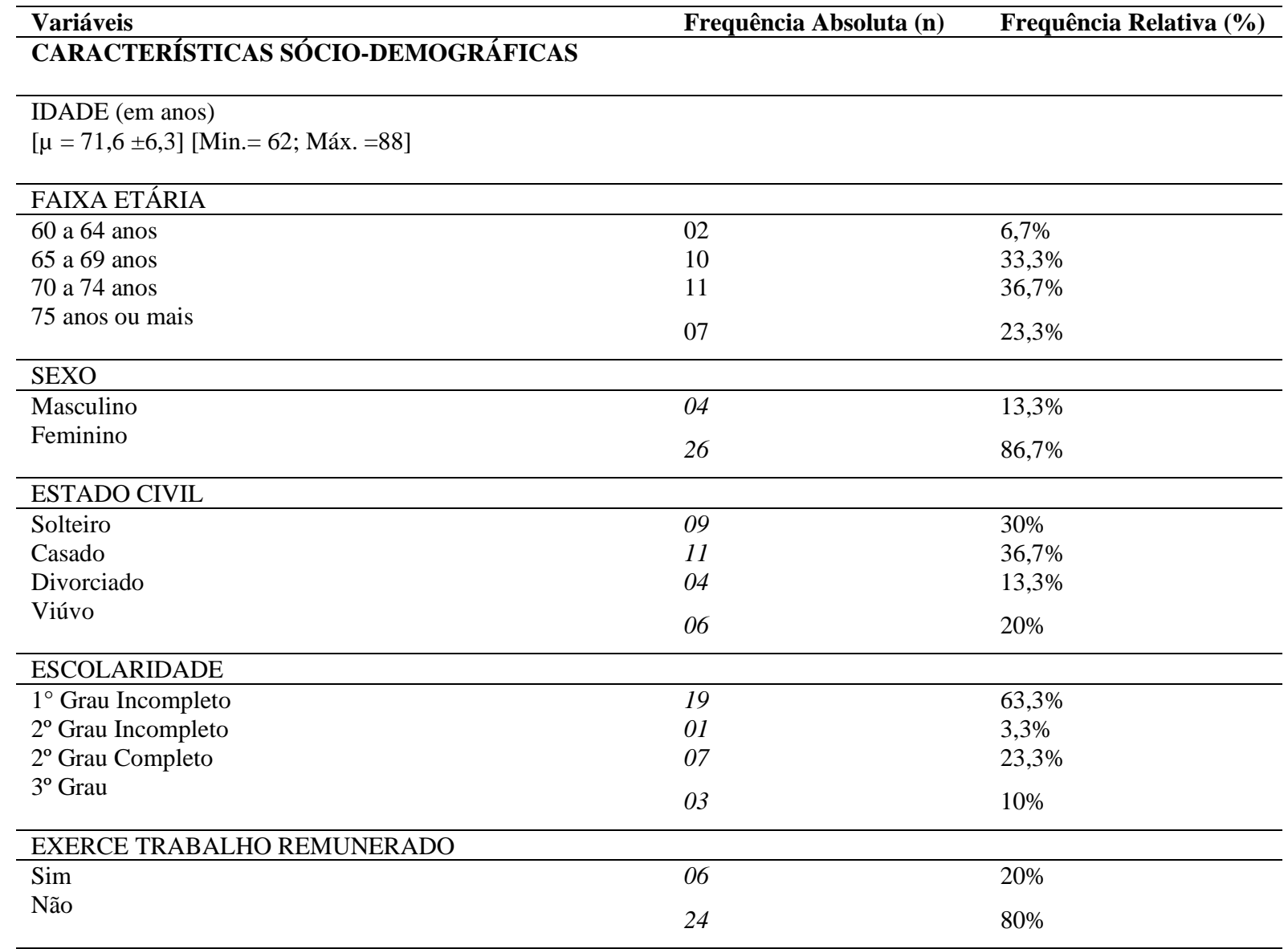

\section{CONDIÇÕES DE SAÚDE}

\begin{tabular}{lll}
\hline QUANTIDADE DE DOENÇAS CRÔNICAS & & \\
\hline 0 & 08 & 26,7 \\
1 & 10 & 43,3 \\
2 ou mais & 12 & 40,0 \\
\hline IMC & 06 & $20 \%$ \\
\hline 22 (Baixo Peso) & 12 & $40 \%$ \\
$\geq 22$ e $<27$ (Eutrófico) & 12 & $40 \%$ \\
& & \\
\hline IRCQ & & $20 \%$ \\
\hline Feminino & \multicolumn{2}{l}{} \\
\hline Baixo Risco $\leq 0,80$ & 6 & \\
\hline
\end{tabular}


Research, Society and Development, v. 10, n. 7, e41110715725, 2021

(CC BY 4.0) | ISSN 2525-3409 | DOI: http://dx.doi.org/10.33448/rsd-v10i7.15725

\begin{tabular}{|c|c|c|}
\hline Risco Moderado 0,81 a 0,85 & 8 & $26,7 \%$ \\
\hline Alto Risco $\geq 0,86$ & 12 & $40 \%$ \\
\hline \multicolumn{3}{|l|}{ Masculino } \\
\hline Baixo Risco $\leq 0,95$ & 1 & $3,3 \%$ \\
\hline Risco Moderado 0,96 a 1,0 & 2 & $6,7 \%$ \\
\hline Alto Risco $\geq 1,0$ & 1 & $3,3 \%$ \\
\hline \multicolumn{3}{|l|}{ PERCEPÇÃO DE SAÚDE } \\
\hline \multirow{4}{*}{$\begin{array}{l}\text { Excelente/ Muito Boa } \\
\text { Boa } \\
\text { Regular } \\
\text { Ruim }\end{array}$} & 08 & $26,7 \%$ \\
\hline & 06 & $20 \%$ \\
\hline & 13 & $43,3 \%$ \\
\hline & 03 & $10 \%$ \\
\hline \multicolumn{3}{|l|}{ HÁBITOS DE VIDA } \\
\hline \multicolumn{3}{|l|}{ USO DE TABACO } \\
\hline \multirow{2}{*}{$\begin{array}{l}\text { Fumante } \\
\text { Não fumante }\end{array}$} & 05 & $16,7 \%$ \\
\hline & 25 & $83,3 \%$ \\
\hline \multicolumn{3}{|l|}{ CONSUMO DE ÁLCOOL } \\
\hline \multirow{2}{*}{$\begin{array}{l}\text { Etilista } \\
\text { Não etilista }\end{array}$} & 02 & $6,7 \%$ \\
\hline & 28 & $93,3 \%$ \\
\hline \multicolumn{3}{|c|}{ PRATICA ATIVIDADE FÍSICA } \\
\hline \multirow{2}{*}{$\begin{array}{l}\text { Sim } \\
\text { Não }\end{array}$} & 22 & $73,3 \%$ \\
\hline & 08 & $26,7 \%$ \\
\hline \multicolumn{3}{|c|}{ TIPO DE ATIVIDADE FISSICA } \\
\hline \multirow{3}{*}{$\begin{array}{l}\text { Lenta / leve } \\
\text { Moderada } \\
\text { Rápida / vigorosa }\end{array}$} & 13 & $43,3 \%$ \\
\hline & 12 & $40 \%$ \\
\hline & 05 & $16,7 \%$ \\
\hline
\end{tabular}

Fonte: Autores.

A Figura 1 apresenta números sobra a presença de comorbidades $40 \%$ dos indivíduos relataram ter duas ou mais doenças crônicas, com destaque para a hipertensão arterial (60\%), osteoporose (30\%), artrite (20\%) e diabetes mellitus $(13,3 \%)$. 
Figura 1: Principais Doenças Crônicas Investigadas. Caicó (RN). 2020.

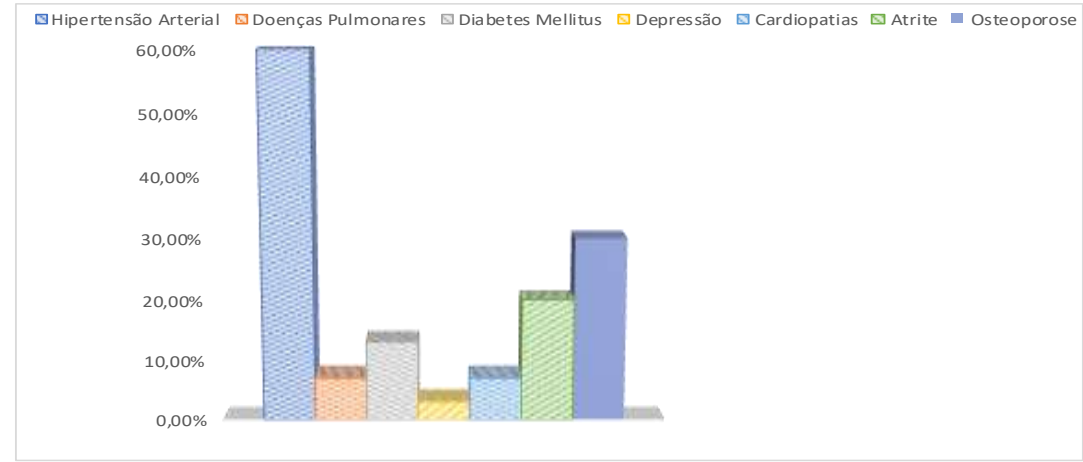

Fonte: Autores.

A Figura 2 apresenta considerações sobre as dimensões das razões motivacionais para a prática de atividade física, analisado através da aplicação do IMPRAF-54, observou-se que a principal razão motivacional registrada foi a dimensão da saúde (32,4\%), seguida pela da sociabilidade (29,7\%), do controle do estresse $(27,8 \%)$, do lazer (25,5\%), da estética (14,7\%) e por fim da competitividade $(11,7 \%)$.

Figura 2: Dimensões motivacionais para prática de atividade físicas em idosos. Caicó (RN). 2020.

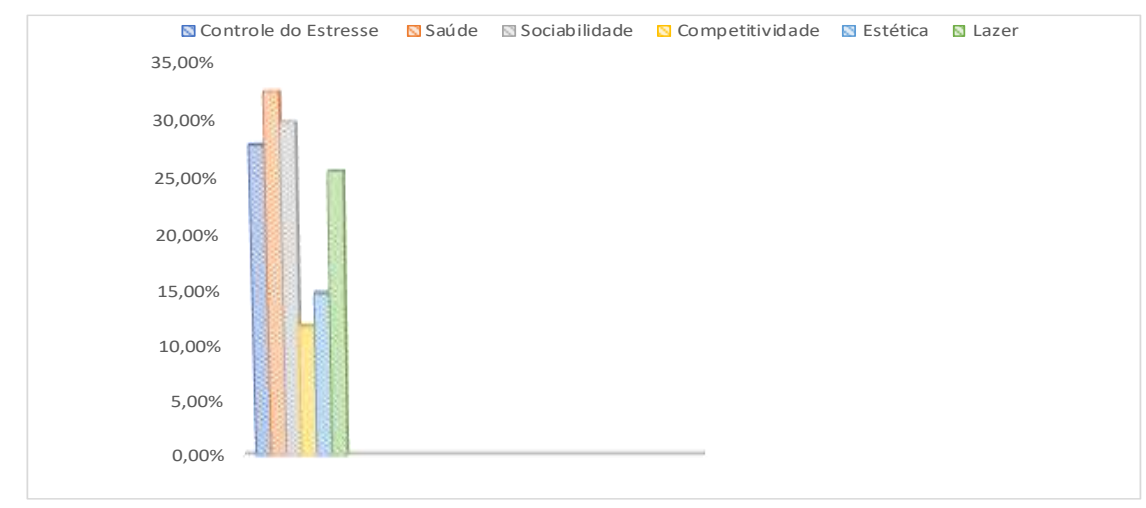

Fonte: Autores.

Para uma melhor compreensão das razões motivacionais para a prática de atividade a Tabela 2 apresenta uma análise de comparação das médias dos escores brutos de todas as dimensões em relação a variáveis independentes de condições de saúde. Evidenciou-se que o escore de motivação para a prática de atividade física aumenta de acordo com a intensidade da atividade física, de forma que atividade física de intensidade moderada à vigorosa apresentou médias maiores de escores para motivação em todas as dimensões, sendo que, apenas na dimensão da "estética" essa diferença foi estatisticamente significativa ( $\mathrm{p}$-valor $<0,05$ ), esse resultado pode estar relacionado ao pequeno número de indivíduos na amostra. 
Research, Society and Development, v. 10, n. 7, e41110715725, 2021

(CC BY 4.0) | ISSN 2525-3409 | DOI: http://dx.doi.org/10.33448/rsd-v10i7.15725

Tabela 2: Comparação da média dos escores de motivação à prática de atividade física com condições de saúde e hábitos de vida. Caicó (RN). 2020.

\begin{tabular}{|c|c|c|c|c|c|c|c|c|c|c|c|c|}
\hline \multirow[t]{2}{*}{ Variáveis } & \multicolumn{2}{|c|}{ Controle do Estresse } & \multicolumn{2}{|c|}{ Saúde } & \multicolumn{2}{|c|}{ Sociabilidade } & \multicolumn{2}{|c|}{ Competitividade } & \multicolumn{2}{|c|}{ Estética } & \multicolumn{2}{|c|}{ Prazer } \\
\hline & $\boldsymbol{\mu} \pm \boldsymbol{\sigma}$ & p-valor & $\mu \pm \sigma$ & p-valor & $\boldsymbol{\mu} \pm \boldsymbol{\sigma}$ & p-valor & $\boldsymbol{\mu} \pm \boldsymbol{\sigma}$ & p-valor & $\boldsymbol{\mu} \pm \boldsymbol{\sigma}$ & p-valor & $\mu \pm \sigma$ & p-valor \\
\hline \multicolumn{13}{|l|}{ IMC } \\
\hline$\leq 22$ (baixo peso) & $25,8 \pm 6,3$ & 0,41 & $29,3 \pm 6,8$ & 0,23 & $27,0 \pm 11,8$ & 0,17 & $14,5 \pm 7,5$ & 0,33 & $12,6 \pm 4,1$ & 0,25 & $23,5 \pm 7,4$ & 0,59 \\
\hline$>22$ e $<27$ (eutrófico) & $26,8 \pm 6,8$ & & $33,2 \pm 4,4$ & & $27,3 \pm 8,1$ & & $12,0 \pm 6,4$ & & $17,1 \pm 7,5$ & & $27,4 \pm 6,1$ & \\
\hline$\geq 27$ (sobrepeso) & $29,5 \pm 5,8$ & & $33,1 \pm 4,1$ & & $33,0 \pm 5,7$ & & $10.2 \pm 4,1$ & & $13,5 \pm 5,3$ & & $24,6 \pm 10,1$ & \\
\hline \multicolumn{13}{|c|}{$\begin{array}{l}\text { TIPO DE ATIVIDADE } \\
\text { FÍSICA }\end{array}$} \\
\hline Lenta / leve & $26,9 \pm 7,2$ & 0,39 & $31,0 \pm 5,8$ & 0,33 & $28,0 \pm 9,2$ & 0,12 & $9,6 \pm 3,3$ & 0,25 & $11,3 \pm 4,6$ & 0,02 & $22,8 \pm 9,8$ & 0,24 \\
\hline Moderada & $27,2 \pm 5,0$ & & $32,8 \pm 3,9$ & & $28,7 \pm 7,6$ & & $13,1 \pm 7,9$ & & $16,2 \pm 5,9$ & & $26,5 \pm 7,2$ & \\
\hline Rápida / vigorosa & $31,4 \pm 6,8$ & & $34,8 \pm 4,3$ & & $36,6 \pm 3,5$ & & $13,6 \pm 4,0$ & & $19,4 \pm 6,3$ & & $29,8 \pm 3,1$ & \\
\hline $\begin{array}{l}\text { QUANTIDADE } \\
\text { DOENÇAS CRÔNICA }\end{array}$ & & & & & & & & & & & & \\
\hline 0 & $26,8 \pm 7,0$ & 0,14 & $31,2 \pm 6,8$ & 0,73 & $30,0 \pm 11,4$ & 0,96 & $14,2 \pm 6,6$ & 0,18 & $17,8 \pm 8,3$ & 0,19 & $24,3 \pm 8,1$ & 0,50 \\
\hline 1 & $25,3 \pm 6,2$ & & $33,1 \pm 4,0$ & & $29,1 \pm 8,7$ & & $9,2 \pm 3,1$ & & $12,7 \pm 4,3$ & & $23,7 \pm 9,4$ & \\
\hline 2 ou mais & $30,5 \pm 5,3$ & & $32,6 \pm 4,3$ & & $30,0 \pm 6,1$ & & $12,1 \pm 6,5$ & & $14,1 \pm 5,4$ & & $27,6 \pm 7,4$ & \\
\hline $\begin{array}{l}\text { PERCEPÇÃO D } \\
\text { SAÚDE }\end{array}$ & & & & & & & & & & & & \\
\hline
\end{tabular}


Research, Society and Development, v. 10, n. 7, e41110715725, 2021

(CC BY 4.0) | ISSN 2525-3409 | DOI: http://dx.doi.org/10.33448/rsd-v10i7.15725

\begin{tabular}{|c|c|c|c|c|c|c|c|c|c|c|c|c|}
\hline Excelente/ Muito Boa & $28,2 \pm 7,8$ & 0,25 & $35,0 \pm 4,2$ & 0,07 & $33,2 \pm 6,4$ & 0,53 & $11,5 \pm 4,2$ & 0,85 & $17,7 \pm 6,4$ & 0,18 & $27,8 \pm 7,8$ & 0,19 \\
\hline Boa & $24,6 \pm 7,5$ & & $28,1 \pm 6,5$ & & $26,8 \pm 7,6$ & & $11,5 \pm 8,1$ & & $10,6 \pm 4,1$ & & $20,1 \pm 7,5$ & \\
\hline Regular & $27,6 \pm 4,6$ & & $32,7 \pm 3,8$ & & $28,9 \pm 10,1$ & & $11,3 \pm 5,9$ & & $14,2 \pm 5,7$ & & $25,0 \pm 8,8$ & \\
\hline Ruim & $33,6 \pm 3,7$ & & $32,6 \pm 2,5$ & & $29,6 \pm 4,9$ & & $14,6 \pm 6,1$ & & $16,3 \pm 8,5$ & & $31,3 \pm 1,1$ & \\
\hline
\end{tabular}

Fonte: Autores. 


\section{Discussão}

Este estudo teve como objetivo investigar as razões motivacionais e os possíveis fatores relacionados à prática de atividade física em pessoas com 60 anos ou mais, atendidos pela atenção primária à saúde do município de Caicó, assim como as características dos idosos quanto a comportamento e fatores que os motivam a praticarem atividade física.

Sabe-se que muitos motivos podem influenciar a inatividade física, dentre eles o gênero, o nível socioeconômico, a escolaridade (Krug, 2012), a autopercepção de saúde (Souza \& Vendrúsculo, 2010), o estado civil, a obesidade, o tabagismo e o álcool (Hirvensalo \& Lintunen, 2011), as condições ambientais, de transporte e de segurança (Cassou et al., 2011), além da imagem negativa do idoso perante a sociedade (Moschny et al., 2011). No presente estudo, foi possível obter uma amostra de 30 indivíduos, com média de idade de 71,3 anos $\pm 6,3$; sendo $86,7 \%$ do sexo feminino e $13,3 \%$ masculino. A maior participação de idosos do sexo feminino pode estar relacionada a feminização do envelhecimento e à maior capacidade de autocuidado por parte das mulheres. Corroboram com este resultado os estudos de Silva e Kraeski (2010), Eiras et al., (2010), Massetto (2011) e Schwingel (2011) que também apresentaram a predominância de mulheres envolvidas em programas de atividades físicas. Por outro lado, López-Benavente et al., (2018) em estudo realizado com idosas, afirmou que a as mulheres tendem a apresentar fatores de desmotivação para a prática de exercício, pois são sobrecarregadas com tarefas domésticas, influenciando uma precária percepção de saúde e pouco tempo livre.

A maioria dos idosos do presente estudo relatou ter união estável, escolaridade inferior ao $1^{\circ}$ grau completo, não exercer atualmente trabalhado remunerado, ser não tabagista e não etilista. Estudos envolvendo participantes de programas de atividade física oferecidas por UBS apresentou características sociodemográficas semelhantes (Carvalho et al., 2016; Lovato et al., 2015; Silva et al., 2011).

Além disso, o presente estudo evidenciou sobrepeso e/ou obesidade em $40 \%$ dos participantes, além de maior proporção de idosos com IRCQ classificado em com risco moderado ou elevado. Tais achados, reforçam as evidências do aumento da prevalência de sobrepeso no Brasil. Segundo Malta et al. (2014), a prevalência do excesso de peso aumentou, em todo território nacional, significativamente, em ambos sexos, em todas as faixas etárias e em todos os níveis de escolaridade. $\mathrm{O}$ estudo de Silva et al. (2018), realizado com 35.214.802 idosos de 60 anos ou mais de idade, também evidenciou a tendência ao aumento da prevalência de sobrepeso e de obesidade nas cinco regiões do país, reforçando a ocorrência da transição nutricional no Brasil.

Apesar do processo de envelhecimento não estar, necessariamente, relacionado a enfermidades, as doenças crônicodegenerativas são frequentemente encontradas entre os idosos, podendo estar ligado aos hábitos da vida diária, alimentação e nível de atividade física. O presente estudo apresentou $40 \%$ dos participantes com 2 doenças crônicas ou mais, sendo a hipertensão arterial a mais relatada; e no tocante à autopercepção da saúde, a maioria considerou a própria saúde como regular ou ruim. Vários estudos nacionais e internacionais trazem achados semelhantes quanto à alta prevalência de comorbidades em idosos e às principais doenças crônicas relatadas (Melo \& Lima, 2018; Pereira et al., 2020; Cavalcanti et al., 2017; Silva et al., 2017; Iepsen \& Silva, 2015). A ocorrência de comorbidade vem sendo estudada no Brasil e é uma questão emergente para a saúde pública brasileira, potencialmente relacionando-a com a autopercepção de saúde (Melo \& Lima, 2018).

Em relação à atividade física, a maioria dos participantes relatou praticar atividade física regularmente de forma leve a moderada. É importante destacar que a prática regular de atividade física auxilia no tratamento das doenças crônicas, reduz o risco de eventos adversos na velhice e de mortes prematuras (Knuthet et al., 2009; Matsudo, 2009; OMS, 2011).

No tocante às razões motivacionais para a prática de atividade física, obteve-se dentre os participantes desta pesquisa que a saúde foi considerada a principal motivação, seguida por sociabilidade, controle do estresse, lazer, estética e competitividade. Além disso, o escore para a prática de atividade física aumentou de acordo com a intensidade da atividade física. O estudo de Faria e Beck (2020), também desenvolvido com idosos e no cenário da APS, observou uma sequência de 
motivação similar para motivação à prática de atividade física sendo saúde, prazer, sociabilidade, estética, controle de estresse e competitividade. Outros estudos nacionais e internacionais trazem achados semelhantes, apontando que idosos de ambos os sexos são predominantemente motivados a prática de atividade física, essencialmente, pelo aspecto saúde, sendo esta a principal referência para o planejamento de aula dos profissionais de educação física (Meurer, 2010; Cavalli et al., 2014; Sjörs et al., 2014; Silva; Castanho; Chiminazzo; Barreira; Fernandes, 2016). A dimensão motivacional da sociabilidade foi o segundo fator mais citado na pesquisa em questão, mostrando-se mais relevante que o controle do estresse. Eime (2013) afirma que as atividades físicas possibilitam interação social no público idoso. Por outro lado, Sjörs (2014) observou que as razões sociais ficaram entre as menos citadas como fator motivacional para atividade física, contrapondo-se aos achados na pesquisa em questão, e de outros que relatam as razões sociais como o fator mais importante (Horne \& Tierney, 2012; Salehi et al., 2010) ou o segundo mais importante (Päivi et al., 2010; Costello et al., 2011). Deve-se considerar que os homens foram sub representados na maioria desses estudos, bem como na pesquisa em questão; além disso, fatores culturais devem ser fortemente considerados no âmbito da socialização. Oliveira et. al. (2019) afirma que fatores como sexo, faixa etária e o nível de atividade física podem interferir na motivação para a prática de atividades física, além do que o avançar da idade parece interferir no nível de autoestima do idoso. Ressalta-se que a prevenção de doenças e o controle do estresse são fatores importantes para as mulheres ao praticar atividade física, enquanto a condição física é um fator de destaque para os idosos ativos fisicamente. A pesquisa de Silva (2018) que também utilizou do IMPRAF-54 para avaliar fatores que fazem com quem idosas fisicamente ativas com idade acima de 60 anos deem início e permaneçam na atividade física, identificou-se que a recomendação médica e o prazer pela atividade física foram uns dos fatores motivacionais para a permanência das idosas nos grupos de ginástica. Os outros fatores motivacionais, como a estética e a competitividade tiveram média menor. Segundo Meurer (2012), a dimensão da estética é um fator de motivação cuja importância diminui com a idade, uma vez que a preocupação se centra em aspectos relacionados à sensação de bem estar e saúde, ao invés da aparência. A competitividade apareceu como o último fator apontado pelos idosos. O estudo de Dantas (2015) também trouxe que a competitividade foi a dimensão que menos motivou os idosos à prática da atividade física. Possivelmente estes não são anseios primordiais dos idosos, uma vez que boa parte destes possivelmente se encontram aposentados, e com objetivos de vida diferentes dos jovens onde a competitividade impera na vida tanto profissional quanto social.

Em estudo realizado com adultos e idosos a respeito da motivação, notou-se que os adultos se mantêm motivados por aspectos multifatoriais, enquanto que os idosos são motivados especificamente pela necessidade de não se sentir só, ou seja, pela sociabilidade, também pela manutenção da saúde e por indicações médicas (Bavoso, Galeote \& Montiel, 2017). Assim, o incentivo a prática regular de atividade física no contexto da UBS e espaços públicos podem contribuir para a adesão de estilo de vida fisicamente ativo, por grande parte da população idosa, uma vez que são espaços gratuitos e acessíveis. Os determinantes motivacionais para a prática da atividade física podem sofrer influências segundo os objetivos de cada indivíduo e o tipo de atividade física, mas, de forma geral, a saúde tem sido considerada como importante motivo para adesão e a socialização um grande influenciador na manutenção da atividade física.

Cabe ressaltar que o presente estudo apresenta limitações. O tempo de coleta de dados e a quantidade de idosos pesquisados devido a eminência da pandemia do COVID-19 que culminou com a suspensão da coleta. O tamanho da amostra foi um fator limitante com importante influência no poder estatístico. A realização do estudo em uma região específica, com amostra por conveniência, inviabilizou que os resultados obtidos sejam extrapolados para outras populações. Análises comparativas mais elaboradas entre os sexos não foram possíveis de serem realizadas devido ao tamanho amostral e a quantidade de participantes do sexo masculino, mas consiste em uma interessante estratégia de análise, considerando-se diferentes fatores motivacionais para homens e mulheres. Estudos futuros, com amostras maiores e delineamentos de pesquisa mais sofisticados, são fundamentais para a obtenção de resultados mais robustos, bem como uma avaliação em longo prazo 
desses fatores motivacionais. Sugere-se que mais estudos com amostras ainda mais expressivas sejam realizados na intenção de compreender as razões motivacionais para prática de atividades físicas em idosos atendidos pela APS, pois entende-se a importância de programas de atividades físicas na vida desta população, pois se trata de uma forma de mantê-los em vida social ativa e saudável tendo em vista as projeções para o aumento de idosos mundialmente, já vividas atualmente.

\section{Conclusão}

A realização desta pesquisa possibilitou caracterizar o perfil sociodemográfico e de saúde de idosos atendidos pela APS da zona leste do município de Caicó (RN). Onde a maioria dos participantes foi do sexo feminino, chamando a atenção para a necessidade de um incentivo maior para a participação do sexo masculino para prática de exercícios físicos e envolvimento nas ações desenvolvidas pelas UBS's, tornando-se importante o direcionamento de atividades e cuidados específicos para os idosos. Os achados deste estudo e a mensuração das razões motivacionais para a prática de atividade física de idosos contribuem na elaboração de ferramentas que atendam às expectativas deste público e auxilie na manutenção em longo prazo desses indivíduos de forma ativa. Além disso, colaboram no planejamento de estratégias de promoção de saúde para idosos, visando um envelhecimento saudável. Sugere-se a inserção e participação de profissionais e acadêmicos dos diversos cursos da área de saúde nas orientações gerais de prevenção, autocuidado e promoção da saúde, devido muitas vezes à carência de uma equipe multidisciplinar para atender as necessidades individuais e coletivas desses idosos, assim como, a realização estudos populacionais sobre a temática para melhor conhecer o perfil da população idosa assim como, a realização estudos populacionais sobre a temática para melhor conhecer o perfil da população idosa, e ainda, que os estudos futuros, relacionados a essa temática, possam trazer uma amostra com um número maior de indivíduos afim de trazer dados ainda concretos acerca das razões motivacionais para prática de exercícios físicos em idosos.

\section{Referências}

Balbinotti, M. A. A. et al. (2011). Motivação à prática regular de atividade física: um estudo exploratório. Estudos de psicologia. 16(1): 99-106.

Bavoso, D., Galeote, 1. \& Montiel, J. M. (2017). Motivação e autoestima relacionada à prática de atividade física em adultos e idosos. Revista Brasileira de Psicologia do Esporte. 7(2), 26-37.

Brasil, MS. (2012). Política nacional de atenção básica.

Brasil, MS. Vigilância alimentar e nutricional. (2004). Orientações básicas para coleta, o processamento, a análise de dados e a informação em serviços de saúde.

Cassou, A. C. N. et al. (2011). Barriers to physical activity among brazilian elderly women from different socioeconomic status: a focus-group study. Journal of physical activity and health. 8, $126-132$.

Cavalcanti, G., Doring, M., Portella, M. R., Bortoluzzi, E. C., Mascarelo, A. \& Delani, M. P. (2017). Multimorbidity associated with polypharmacy and negative self-perception of health. Revista brasileira geriatria e gerontologia. 20(5):634-642.

Cavalli, A. S., Garcia, G. P., Ricardo, A. C., Ribeiro, J. A. B. \& Cavalli, M. O. (2019). Fatores motivacionais de idosos participantes em projetos sociais universitários de educação física. Estudo interdisciplinar envelhecimento. Porto Alegre. Edição Especial, v. 24, p. 11-25.

Cavalli, A. S., Pogorzelski, 1. D. V, Domingues, M. R., Afonso, M. D. R., Ribeiro, J. A. B. \& Cavalli, M. O. (2014). Motivation of elderly people to engage in physical exercising: a comparative study between two university-based programs Brazil and Portugal. Revista brasileira geriatria e gerontologia. 17(2):25564.

Carvalho, F. F. B. \& Nogueira, J. A. D. (2016). Práticas corporais e atividades físicas na perspectiva da promoção da saúde na atenção básica. Ciência \& Saúde Coletiva. 21(6):1829-1838.

Charro, M. A., Figueira Júnior, A. \& Allegretti, J. G. (2020). Tratado de musculação. Ed. Phorte.

Costello, E., Kafchinski, M., Vrazel, J. \& Sullivan P. (2011). Motivators, barriers, and beliefs regarding physical activity in an older adult population. Journal geriatria physicus ther. 34(3):138-47.

Dantas, R. et al. (2015). Fatores motivacionais de idosos praticantes do método pilates. Revista brasileira em promoção da saúde. 28(2). 
Eiras, S. B., Silva, W. H. A., Souza, D. L. \& Vendruscolo, R. (2010). Fatores de adesão e manutenção da prática de atividade física por parte de idosos. Revista brasileira de ciências do esporte. 31(2):75-89.

Eime, R. M. et al. (2013). A systematic review of the psychologicaland social benefits of participation in sport for chil-dren and adolescents: informing development of a con-ceptual model of health through sport. The International Journal of Behavioral Nutrition and Physical Activity. 10:98.

Farias Júnior, A. (2015). Atividade física e envelhecimento humano. H.P. Comunicação.

Fleck, S. J. \& Kraemer, W. J. (2017). Fundamentos do treinamento de força muscular. (4a ed.), ARTMED.

Hirvensalo, M. \& Lintunen, T. (2011). Life-course perspective for physical activity and sports participation. European Revew of Aging and Physical Activity. $8,13-22$.

Horne, M. \& Tierney, S. (2012). What are the barriers and facilitators to exercise and physical activity uptake and adherence among south asian older adults: a systematic review of qualitative studies. Prev Med. 55(4):276-84.

IBGE. (2020). Estimativas populacionais para municípios brasileiros: http://www.ibge.gov.br.

IBGE. (2019). População idosa. http://www.ibge.gov.br.2019.

Iepsen, A. M., Silva \& M. C. (2015). Perfil dos frequentadores das academias ao ar livre da cidade de Pelotas-RS. Revista Brasileira de Atividade Física e Saúde. 20(4):413-24.

Knuth, A. G., Bielemann, R. M., Silva, S. G., Borges, T. T. \& Del Duca, G. F. (2009). Conhecimento de adultos sobre o papel da atividade física na prevenção e tratamento de diabetes e hipertensão: estudo de base populacional no sul do Brasil. Caderno de saúde pública. 25(3):513-20.

Krug, R. R. (2012). Idosas longevas inativas fisicamente: percepção das barreiras e facilitadores para a prática da atividade física. [Dissertação mestrado em ciências do movimento humano]. Florianópolis: UESC.

Lima, A. P. \& Delgado, E. I. (2017). A melhor idade do Brasil: aspectos biopsicossociais decorrentes do processo de envelhecimento. ACTA Brasileira do Movimento Humano.

López-benavente, Y., Arnau-sánchez, J., Ros-sánchez, T., Lidón-cerezuela, M. B., Serrano-noguera, A. \& Medina-abellán, M. D. (2018). Difficulties and motivations for physical exercise in women older than 65 years. A qualitative study. Revista Latino-americana de Enfermagem. 26,1 -10.

Lovato, S. S., Loch, M. R, Gonzávez, A. D \& Lopes, M. L. S. (2015). Assiduidade a programas de atividade física oferecidas por unidades básicas de saúde: o discurso de participantes muito e pouco assíduos. Revista Brasileira de Atividade Física e Saúde. 20(2):184-192.

Malta, D. C., Andrade, S. C., Claro, R. M., Bernal, R. T. I. \& Monteiro, R. C. A. (2014). Evolução anual da prevalência de excesso de peso e obesidade em adultos nas capitais dos 26 estados brasileiros e no distrito federal entre 2006 e 2012. Revista Brasileira de Epidemiologia. $2014 ; 267-76$.

Massetto, S. T. (2011). Manutenção da prática de atividades aquáticas: tempo de prática e alterações dos principais motivos, na percepção dos idosos. Revista Mackenzie de Educação Física e Esporte. 10, 76-88.

Matsudo, S. M. (2009). Envelhecimento, atividade física e saúde. Bol. Inst. Saúde. 47.

Melo, L. A. \& Lima, K. C. (2018). Prevalência e fatores associados a multimorbidades em idosos brasileiros. Ciência \& Saúde Coletiva. $25(10): 3869-3877$.

Meurer, S. T., Benedetti, T. R. B. \& Mazo, G. Z. (2012). Fatores motivacionais de idosos praticantes de exercícios físicos: um estudo baseado na teoria da autodeterminação. Estudos de Psicologia. mai./ago.; 17(2), 299-303.

Meurer, S. T. (2010). Motivação para a prática de exercícios físicos, autoestima e aptidão funcional de idosos: interpretações baseadas na teoria da autodeterminação. 2010. 140 f. [Dissertação de mestrado em Educação Física]. Florianópolis: UFSC.

Moschny, A., Platen, P., Klaassen-mielke, R., Trampisch, U. \& Hinrichs, T. (2011). Barriers to physical activity in older adults in germany: a cross- -sectional study. Int J Behav Nutr Phys Act. 8(1):121.

OEA. (2015). Convenção Interamericana sobre a Proteção dos Direitos Humanos dos Idosos. Washington: OEA.

Oliveira, D., Ladeira, A., Giacomin, I., Pivetta, N., Antunes, M., Batista, R. \& Nascimento Júnior, J. (2019). Depressão, autoestima e motivação de idosos para a prática de exercícios físicos. Psicologia, Saúde \& Doenças. 20(3), 803-812.

OPAS. (2019). Plano de ação sobre a Saúde dos Idosos, incluindo o envelhecimento ativo e saudável: Relatório Final. OPAS.

Päivi, M., Mirja, H. \& Terttu, P. (2010). Changes in physical activity involvement and attitude to physical activity in a 16- year follow-up study among the elderly. J Aging Res. 174290.

Pereira, B. P., Bortolotto, C. C., Tomasi, E., Gonzalez, M. C., Gomes, A. P., Gonçalves, H. \& Bielemann, R. M. (2020). Consumo alimentar e multimorbidade entre idosos não institucionalizados de pelotas, 2014: estudo transversal. Epidemiol. Serv. Saúde. Brasília: 29(3): e 2019050.

Salehi, L., Eftekhar, H., Mohammad, K., Taghdisi, M. H. \& Shojaeizadeh, D. (2010). Physical activity among a sample of iranians aged over 60 years: an application of the transtheoretical model. Arch Iran Med. 13(6):528-36.

Schwingel, V. (2011). Aderência de idosos no projeto de hidroginástica do projetar. 2011. 57f. [Monografia Bacharelado em Educação Física]. UFRS 
Research, Society and Development, v. 10, n. 7, e41110715725, 2021

Silva, F. C. D. (2018). Os efeitos da hidroginástica na resistência muscular localizada dos membros superiores em idosas fisicamente ativas com idade acima de 60 anos [Tese Doutorado]. UFRN.

Silva, H. O., Carvalho, M. J. A. D., Lima, F. E. L. L. \& Rodrigues, L. V. (2011). Perfil epidemiológico de idosos frequentadores de grupos de convivência no município de Iguatu (CE). Revista Brasileira de Geriatria e Gerontologia. 14(1): 123-33.

Silva, L. C. \& Kraeski, D. (2010). Motivos de adesão e permanência em programas de atividade física por idosos. 2006. 10f. [Artigo Licenciatura em Educação Física - Centro de Ciências da Saúde e do Esporte]. UESC.

Silva, N. C., Castanho, K. F., Chiminazzo, J. G. C., Barreira, J. \& Fernandes, P. T. (2017). Fatores motivacionais relacionados à prática de atividades físicas em idosos. Psicologia em Estudo. 21(4), 677-685.

Silva, V. S., Souza, I., Silva, D. A. S., Barbosa, A. R. \& Fonseca, M. J. M. (2018). Evolução e associação do IMC entre variáveis sociodemográficas e de condições de vida em idosos do Brasil: 2002/03-2008/09. Ciência \& Saúde Coletiva. 23(3):891-901.

Sjörs, C., Bonn, S. E., Lagerros, Y. T., Sjölander, A. \& Bälter, K. (2014). Perceived reasons, incentives, and barriers to physical activity in swedish elderly men. Interact J Med Res. 3(4):e15.

Souza, D. L. \& Vendrusculo, R. (2010). Fatores determinantes para a continuidade da participação de idosos em programas de atividade física: a experiência dos participantes do projeto "sem fronteiras". Revista Brasileira de Educação Física e Esporte. v. 24, n. 1.

Veras, R. P. \& Oliveira, M. (2018). Envelhecer no Brasil: a construção de um modelo de cuidado. Ciência \& Saúde Coletiva. 23(6):1929-1936.

WHO. (2011). Global recommendations on physical activity for Health.

WHO. (2008). Waist circumference and waist-hip ratio. Report of a WHO expert consultation.

WHO. (2000). Obesity: preventing and managing the global epidemic. Report of a WHO Consultation. World Health Organization. 